Article

\title{
Numerical and Experimental Study of a Vortex Structure and Energy Loss in a Novel Self-Priming Pump
}

\author{
Hao Chang ${ }^{1,2}$, Ramesh K. Agarwal ${ }^{2, *}$, Wei Li ${ }^{1,2} \mathbb{D}$, Ling Zhou ${ }^{1,2} \mathbb{D}$ and Weidong Shi ${ }^{3, *}$ \\ 1 Research Center of Fluid Machinery Engineering and Technology, Jiangsu University, Zhenjiang 212013, \\ China; haochang@wustl.edu (H.C.); $1000003263 @ u j s . e d u . c n$ (W.L.); lingzhoo@hotmail.com (L.Z.) \\ 2 Department of Mechanical Engineering and Materials Science, Washington University in St. Louis, \\ St. Louis, MO 63130, USA \\ 3 School of Mechanical Engineering, Nantong University, Nantong 226019, China \\ * Correspondence: rka@wustl.edu (R.K.A.); wdshi@ujs.edu.cn (W.S.)
}

Received: 28 August 2019; Accepted: 27 September 2019; Published: 4 October 2019

\begin{abstract}
The self-priming pump as an essential energy conversion equipment is widely used in hydropower and thermal power plants. The energy losses in the internal flow passage of the pump directly affect its work efficiency. Therefore, it is important to improve the internal flow characteristic of the pump. In the present work, a novel self-priming pump which starts without water is proposed; this pump can reduce the energy consumption as well as the time needed to start its operation. The spatial structure of the vortices in the pump is investigated by employing the $Q$ criterion with the numerical solution of the vorticity transport equation. Based on the morphology, the vortices can be separated into three categories: Trailing Edge Vortex (TEV), Leading Edge Vortex (LEV) and Gap Leakage Vortex (GLV). Generally, the morphology of the TEV is more disorderly than that of LEV and GLV, and the intensity of TEV is significantly higher than that of the other two vortices. To determine the magnitude and distribution of energy loss in the pump, entropy production analysis is employed to study the influence of blade thickness on energy characteristics of the pump. It is found that with an increase in the flow rate, the location of energy loss transfers from the trailing edge to the leading edge of the blade, and viscous entropy production (VEP) and turbulence entropy production (TEP) are the dominant factors which influence the energy conversion in the pump. More importantly, employing the blade with a thin leading edge and a thick trailing edge can not only significantly reduce the impact of incoming flow under over-load condition (flow rate higher than the design condition) but can also increase the efficiency of the pump. Thus, an increase in thickness of the blade from the leading edge to the trailing edge is beneficial for improving the pump performance. The results of this paper can be helpful in providing guidelines for reducing the energy loss and in improving the performance of a self-priming pump.
\end{abstract}

Keywords: vortex structure; energy loss; entropy production; self-priming pump

\section{Introduction}

The self-priming pump as an essential form of energy conversion equipment is widely used in hydropower and thermal power plants. The energy loss in the internal flow passage of the pump directly affects its work efficiency. Therefore, it is important to improve its internal flow characteristics by improving the design of its blades [1]. In recent years, considerable research has been conducted on improving the energy characteristics of the pump by modifying its blade profile. Wang et al. [2] proposed an innovative impeller with forward-curving blade by numerically and experimentally investigating the relationship between the energy loss in the impeller and the blade inlet angle, and 
obtained an optimal range for the inlet angles. Elyamin et al. [3] investigated the influence of the number of blades on the energy performance by using numerical simulations and concluded that the increase in number of blades can weaken the secondary flow, resulting in a reduction in the energy loss of the impeller. Shao et al. [4] used high-speed photography to study the internal characteristics of the pump with an impeller with different geometrical structures; they found that the complex vortex structures at low flow rates (partial-load conditions) can be eliminated by decreasing the outlet angle and width of the blade. Nejad and Riasi [5] conducted an experimental test to analyze the relationship between the blade profile and the energy performance; the blades were manufactured from straight to curved profiles with uniform inlet and outlet angles. They showed that reducing the blade angle is beneficial for improving the pump efficiency. Han et al. [6] selected the blade wrap angle and blade exit angle as an analysis variable in their numerical and experimental investigation to optimize the pump performance; they considered the effect of blade parameters on the distribution of turbulent kinetic energy, which affects the performance of the pump. Jeon et al. [7] designed a novel S-shape blade by employing the design of experiment (DOE) and the response surface method (RSM), and showed that the blade's efficiency was enhanced by 3\% compared to under the original model. Nejad at el. [8] modified the blade profile into a bucket shape and studied the influence of blade angle, chord, and other parameters on the hydraulic performance; they concluded that the maximum efficiency of the pump can be improved by employing an optimal combination of these parameters. Wang et al. [9-11] pointed out the factors influencing the energy loss in a multistage centrifugal pump and analyzed the hydraulic loss caused by surface roughness of the impeller shroud experimentally.

However, while most of the investigations noted above studied the effect of varying the number of blades, inlet angle, outlet width, wrap angle, and riding position on the energy efficiency of a pump; on the effect of modification of blade thickness on pump performance has hardly been studied. Furthermore, the influence of unstable vortex structures on the internal flow passage of the pump and their contribution to energy loss has not been studied. In addition, the overall energy loss analysis has only been conducted using the conventional approach by determining the hydraulic efficiency or turbulent kinetic energy, which does not provide the main details of energy loss, namely its location, sources, and type. In the present work, entropy production analysis is introduced to identify the sources, locations, and reasons for energy loss/dissipation. Wang [12] investigated the cavitation performance of the LNG cryogenic pump; they showed that the evolution and degradation that occur due to cavitation can be determined by applying the entropy production model, and then verified the results of their analysis through an experiment. Hou et al. [13] combined the orthogonal design with entropy production analysis to optimize the geometric parameters of the pump; the optimal design was obtained which improved the energy performance of the pump. Gu et al. [14] analyzed the influence of the clocking positions on the hydraulic loss and obtained the energy loss distribution in the pump by employing entropy production analysis. Li et al. [15] introduced the concept of wall entropy production in their investigation; they pointed out that a large deviation in energy loss occurs with respect to experiments without considering the losses that occur due to the wall effect. Thus, entropy production analysis is used in this paper to accurately evaluate the energy loss that occurs in the pump.

In this paper, a novel self-priming pump start without water has been proposed and studied by using the $Q$ criterion with vorticity transport equation and employing the entropy production analysis for quantification of energy losses. The vortex structures inside and energy characteristics of the pump with different blade thicknesses are systematically investigated by using numerical simulations. Numerical simulations are validated for one of the most promising blade thickness distribution in terms of pump head and efficiency by conducting an experiment. Finally, guidelines for obtaining a good thickness distribution for the blades are provided for improving the flow characteristics and performance of the pump. 


\section{Structure of Novel Self-Priming Pump}

Traditional centrifugal pumps must fill the pump with water before starting, which takes lot of time and results in energy loss. Therefore, a novel self-priming pump is proposed in this paper which can start without water. Figure 1 shows the structure of the proposed novel self-priming pump; it shows the inlet, self-priming system, compressor, pump, outlet and outlet valve of the novel self-priming pump. The specifications of the pump are listed in Table 1.

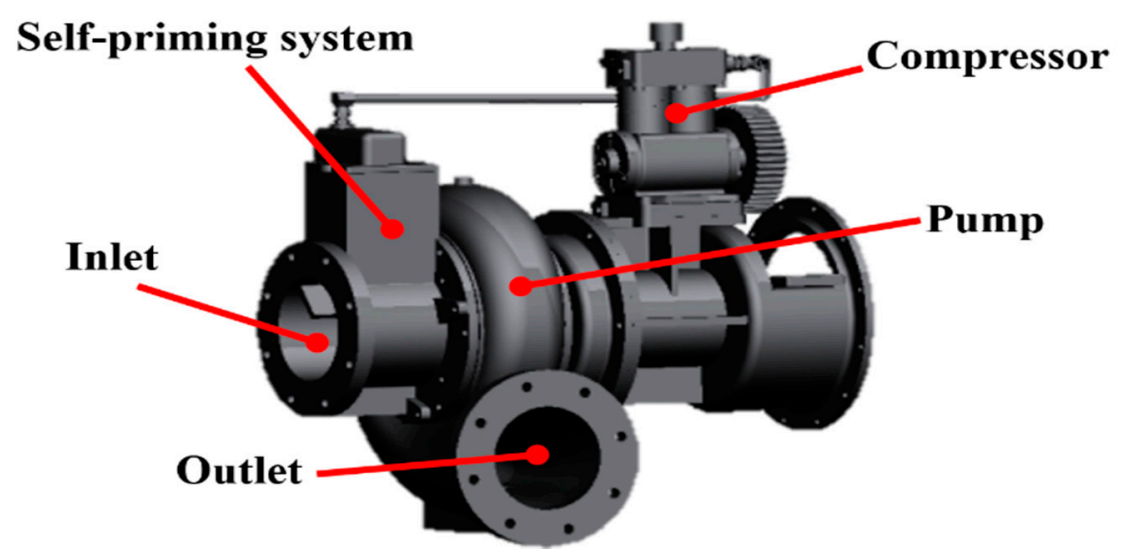

Figure 1. The structure and components of the pump.

Table 1. Specifications of the pump.

\begin{tabular}{ccc}
\hline Parameter & Symbol & Value \\
\hline Head & $H$ & $45 \mathrm{~m}$ \\
Design flow condition & $Q_{\mathrm{d}}$ & $500 \mathrm{~m}^{3} / \mathrm{h}$ \\
Rotation speed & $n$ & $2200 \mathrm{r} / \mathrm{min}$ \\
Specific speed & $n_{\mathrm{s}}$ & 172 \\
Impeller inlet diameter & $D_{1}$ & $200 \mathrm{~mm}$ \\
Impeller outlet diameter & $D_{2}$ & $280 \mathrm{~mm}$ \\
Blade numbers & $Z$ & 6 \\
Blade wrap angles & $\phi$ & $120^{\circ}$ \\
Volute inlet width & $b_{1}$ & $80 \mathrm{~mm}$ \\
Volute outlet diameter & $D_{4}$ & $200 \mathrm{~mm}$ \\
\hline
\end{tabular}

As shown in Figure 1, the self-priming system is installed immediately after the inlet and consists of an induction chamber, filter screen, valve seat, ball valve, plate, valve chamber, intake tube, nozzle 1 , joint, and nozzle 2, as shown in Figure 2. The inlet tube extends into the water before the pump starts, and then the compressor starts running with the operation of the pump. The jet-stream created in the compressor is discharged into nozzle 1 through the intake tube. The inner structure of nozzle 1 first shrinks and then smoothly expands, which results in a high-velocity jet-stream being created through nozzle 1.

A joint is installed between the nozzle 1 and nozzle 2 and is connected with the valve chamber. Hence, the high-velocity jet-stream passes through the joint and nozzle 2 exhausts directly into the atmosphere. At the same time, the internal pressure of the joint is continuously decreasing which leads to pushing the air in the valve box into the joint and eventually discharging it from nozzle 2 . Accordingly, the pressure in the valve box gradually reduces. When the gravity of the ball valve is less than the pressure difference experienced by it, the ball valve is lifted from the valve seat and the air in the induction chamber flows into the valve box and eventually drains into the atmosphere. Furthermore, since the induction chamber is installed at the inlet, the air in the pump exhausts into the atmosphere. Ultimately, water flows into the pump along the inlet tube and the pump is filled with water, which achieves the pump start without water. When the process of self-priming is finished, the ball valve is merged in the water. Under the effect of gravity, it returns to the valve seat and closes the 
connection between the induction chamber and the valve box, avoiding the energy loss resulting from the water discharging into the atmosphere.

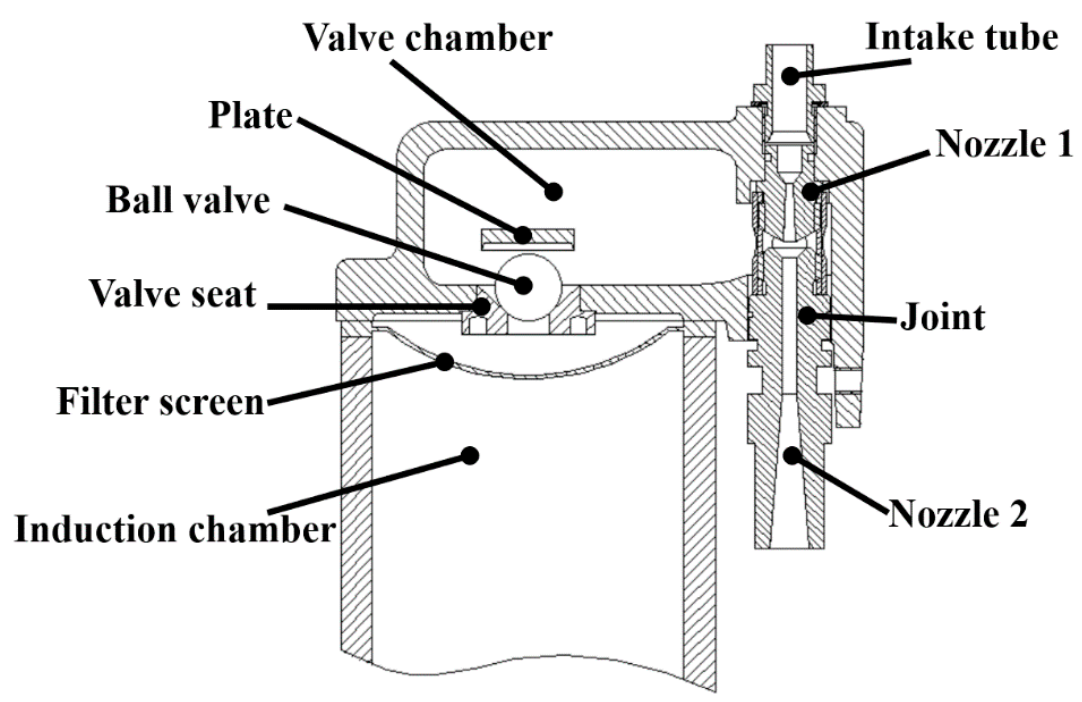

Figure 2. Structure of the self-priming system.

The outlet valve is installed on the outlet and is connected to the atmosphere in order to prevent the air flow into the pump. A rubber plate is pressed against the inclined exit of the outlet tube. When the air inside the pump is drained, the pump is filled with water and under the effect of pressure, the rubber plate separates from the exit of the outlet tube, and thus the self-priming of the pump is completed. The pump thus accomplishes the waterless start and can deliver water normally. The structure of the outlet valve as shown in the Figure 3.

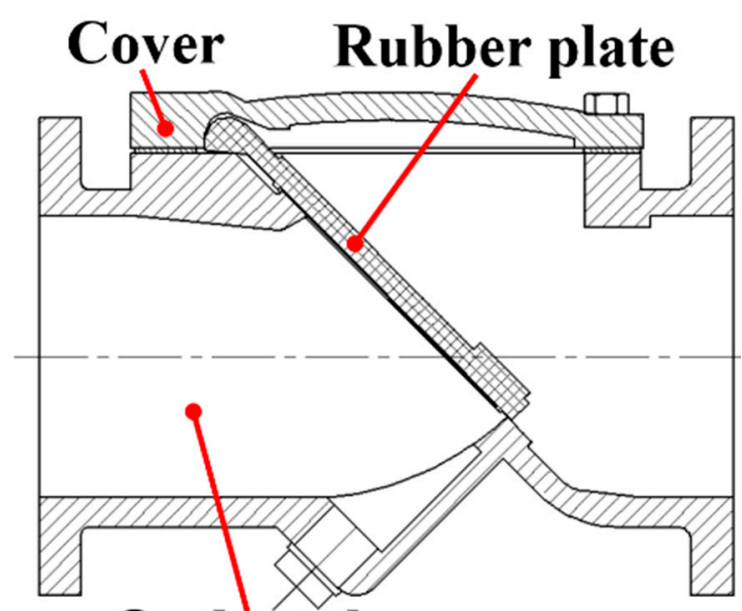

Outlet tube

Figure 3. Structure of the outlet valve.

The geometric parameters of the blade cannot only decide the work capability of the pump, but can also affect the internal flow characteristic In line with our previous investigation, four different blade thickness distributions are proposed [16]; these are shown in Table 2. The 3D models of four blade thickness distribution schemes is shown in the Figure 4. Scheme 1 and scheme 2 employ the uniform thin and thick blade respectively, while the scheme 3 and scheme 4 employ the non-uniform increasing and decreasing blade thickness distribution respectively. 
Table 2. Blades with different thickness distributions.

\begin{tabular}{cccc}
\hline Scheme & $\begin{array}{c}\text { Thickness of Leading } \\
\text { Edge }(\mathbf{m m})\end{array}$ & $\begin{array}{c}\text { Thickness of Middle } \\
\text { Part }(\mathbf{m m})\end{array}$ & $\begin{array}{c}\text { Thickness of Trailing } \\
\text { Edge }(\mathbf{m m})\end{array}$ \\
\hline 1 & 3 & 3 & 3 \\
2 & 6 & 6 & 6 \\
3 & 3 & 6 & 6 \\
4 & 6 & 6 & 3 \\
\hline
\end{tabular}

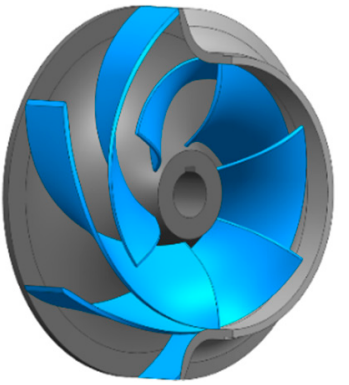

Scheme 1

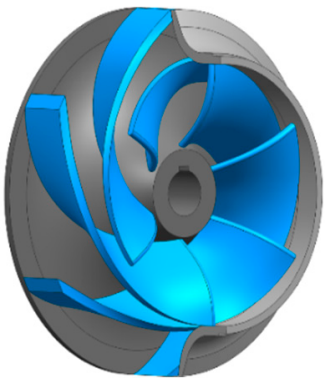

Scheme 2

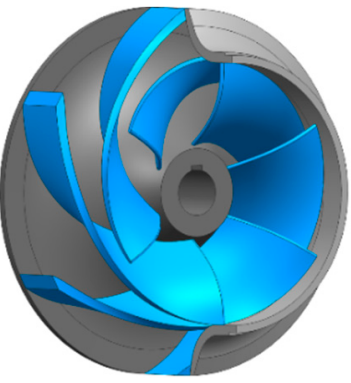

Scheme 3

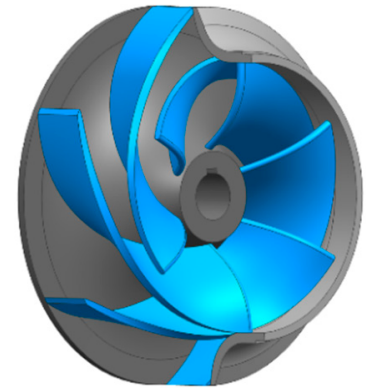

Scheme 4

Figure 4. The 3D models based on four blade thickness distribution schemes given in Table 2.

\section{Computational Model and Numerical Method}

\subsection{Computational Domain}

Figure 5 shows the computational domains of s novel self-priming pump including the outlet, volute, impeller, self-priming system, and inlet. In addition, the stable inflow and outflow can be obtained by extending the inlet and outlet, respectively.

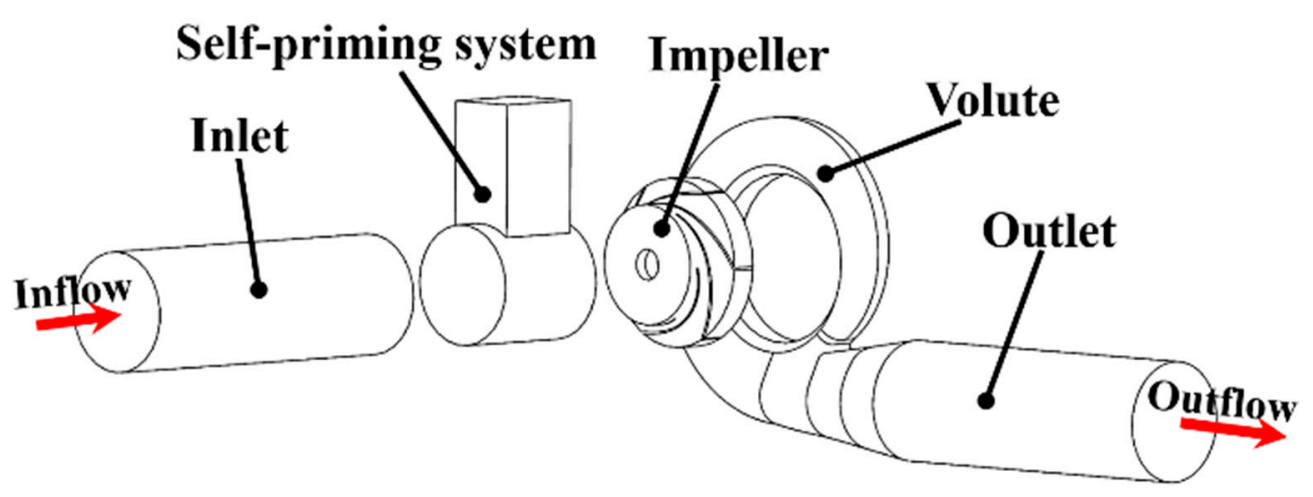

Figure 5. The computational domains.

\subsection{Grid Generation}

The computational domains of the self-priming pump are discretized into a hexahedral structure by employing the ICEM-CFD (The Integrated Computer Engineering and Manufacturing code for Computational Fluid Dynamics). The leading edge of the blade and tongue of the volute are refined to capture the details of the internal flow characters, as shown in Figures 6 and 7.

Since the grid resolution can significantly affect the accuracy of the simulation, grid sensitivity analysis was conducted. As shown in Figure 8, the number of grid elements was varied from $1.2 \times 10^{6}$ to $5.3 \times 10^{6}$ to evaluate the grid independence of the solution. When the number of grid elements exceeds $2.6 \times 10^{6}$, the deviation in pump efficiency and pump head are reduced to less than $1 \%$ by further increasing the number of grid elements. Therefore, considering the computational efficiency of 
the simulation, the grid elements are set as $2.6 \times 10^{6}$ in all simulations reported in this paper and $y+$ is less than 60 . The number of grid elements for the impeller, volute, outlet, inlet, and self-priming system are $478,998,720,480504,000,696,000$ and 272,232 , respectively.

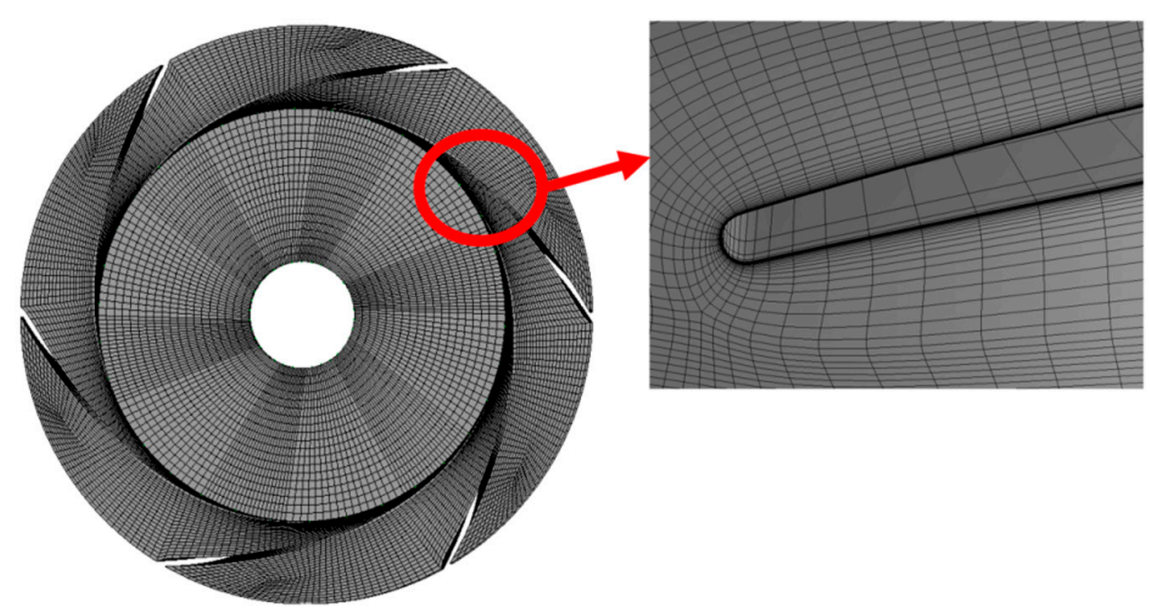

Figure 6. Grid distribution in an impeller.

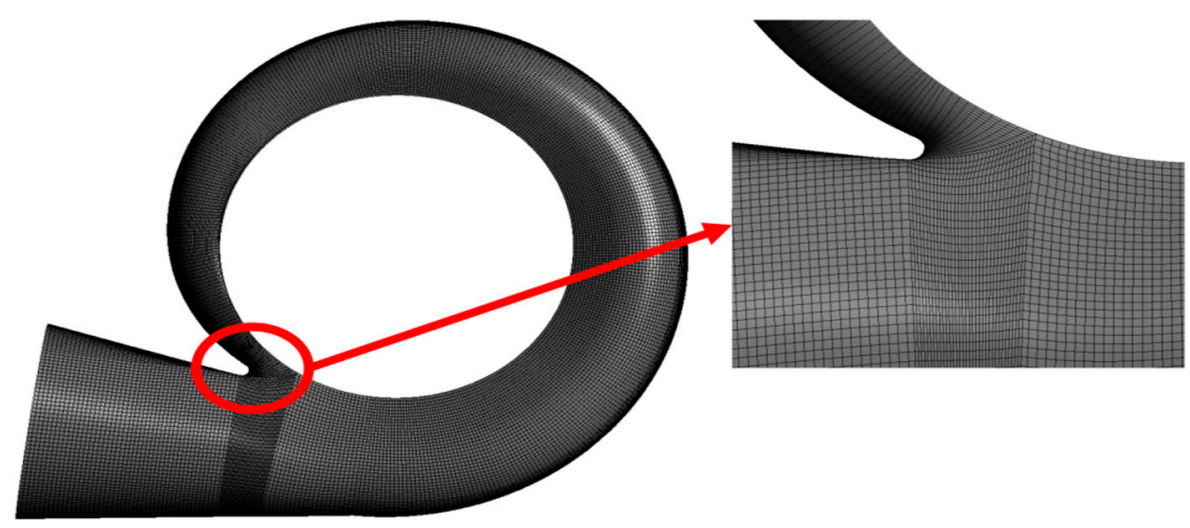

Figure 7. Grid distribution in the volute.

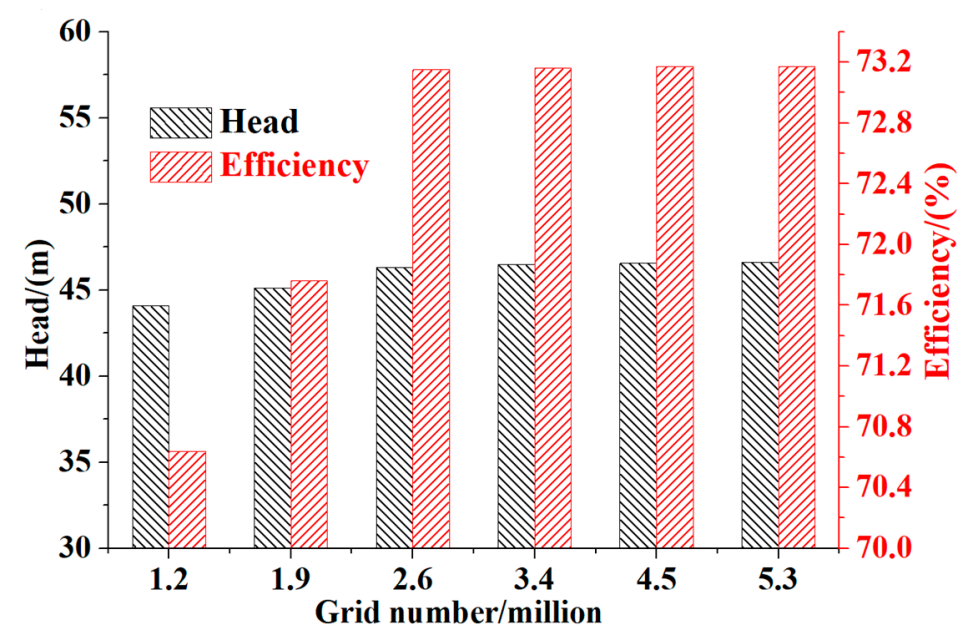

Figure 8. Sensitivity analysis of the number of grid elements used for the solution. 


\subsection{Calculation Method}

In this paper, the SST k- $\omega$ turbulence model with Reynolds-Averaged Navier-Stokes (RANS) equations is employed for developing the numerical solution using the commercial CFD solver ANSYS CFX 17.1. The fluid medium is incompressible water at $25^{\circ} \mathrm{C}$. Considering the impeller as a rotating domain, the surfaces at the inlet and outlet of the impeller are set as the frozen rotor interfaces. Figure 9 shows the sensitivity of the calculations to time steps. The step sizes of 1/120 $\mathrm{T}\left(2.27 \times 10^{-4} \mathrm{~s}\right), 1 / 60 \mathrm{~T}$ $\left(4.54 \times 10^{-4} \mathrm{~s}\right)$ and $1 / 30 \mathrm{~T}\left(9.08 \times 10^{-4} \mathrm{~s}\right)$ are selected as time steps in the investigation. By comparing the pressure at the outlet with different time steps, it was found that small variations exist among the results when using different time steps. Based on this study on the influence of time steps on the solution, $1 / 60 \mathrm{~T}\left(4.54 \times 10^{-4} \mathrm{~s}\right)$ is selected as the time step for computational efficiency without compromising accuracy $[17,18]$. The numerical setup is described in Table 3.

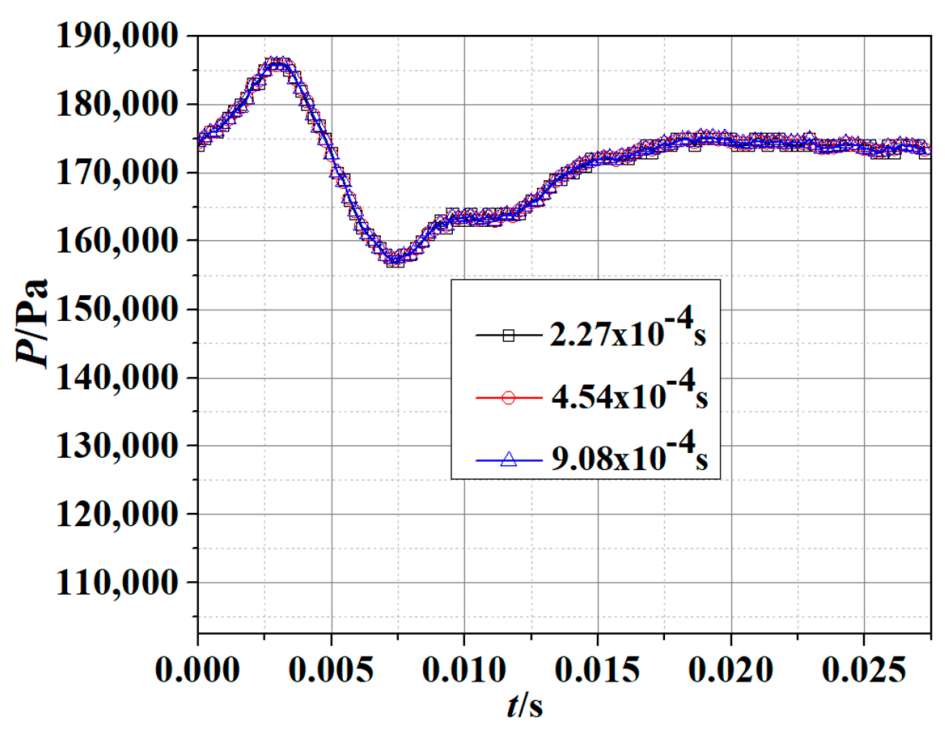

Figure 9. Analysis of the sensitivity of time steps in the numerical simulation.

Table 3. The numerical setup.

\begin{tabular}{cc}
\hline CFD Software & ANSYS CFX 17.1 \\
\hline Turbulence model & SST k- $\omega$ \\
Flow medium & Water at $25^{\circ} \mathrm{C}$ \\
Inlet boundary condition & Total pressure $(1 \mathrm{~atm})$ \\
Outlet boundary condition & Mass flow \\
Wall roughness & $50 \mu \mathrm{m}$ \\
Steady state & Frozen rotor \\
Transient state & Transient rotor-stator \\
Advection scheme & High resolution \\
RMS residual & 0.00001 \\
Transient calculation time & $0.2 \mathrm{~s}$ \\
Time step & $4.54 \times 10^{-4} \mathrm{~s}$ \\
Turbulence intensity & $5 \%$ \\
\hline
\end{tabular}

\section{Results and Discussion}

\subsection{Analysis of the Vortex Structure}

Due to the complex structure of the novel self-priming pump, several types of vortices are generated in the internal flow field, namely the secondary flow vortex, reflux vortex, and flow separation vortex. Therefore, to reduce the influence of the vortices on the hydraulic performance of the pump, the $Q$ criterion is employed to investigate the morphology and structure of vortices. Figure 10 shows the 
vortices in the impeller and the pressure distributions on the impeller for the four different blade thickness distributions given in Table 2. The equations of the $Q$ criterion can be written as follows [19]:

$$
\lambda^{3}+P \lambda^{2}+Q \lambda+R=0 .
$$

Considering water as an incompressible medium, the equivalent equations are:

$$
\begin{gathered}
P=S_{i i}=0 \\
Q=\left(\Omega_{i j} \Omega_{j i}-S_{i j} S_{j i}\right) / 2 \\
R=-\left(S_{i j} S_{j k} S_{k i}+3 \Omega_{i j} \Omega_{j k} S_{k i}\right) / 3
\end{gathered}
$$

and

$$
\begin{aligned}
S_{i j} & =\left(\partial u_{i} / \partial x_{j}+\partial u_{j} / \partial x_{i}\right) / 2 \\
\Omega_{i j} & =\left(\partial u_{i} / \partial x_{j}-\partial u_{j} / \partial x_{i}\right) / 2 .
\end{aligned}
$$

In Equation (1), $P, Q$, and $R$ represent the invariants of velocity gradient tensor. The $Q$ criterion was proposed based on the second invariant of velocity gradient tensor. $S_{i j}$ and $\Omega_{i j}$ represent tensors of strain rate and vorticity, respectively and reflect the deformation and rotational motion. Figure 10 depicts the three-dimensional (3D) vortex structures with blade thickness distribution given in Table 2 under various flow conditions. According to the morphology and structure of vortices, they can be separated into three categories, namely the leading edge vortex (LEV), trailing edge vortex (TEV), and gap leakage vortex (GLV).

$[\mathrm{Pa}]$
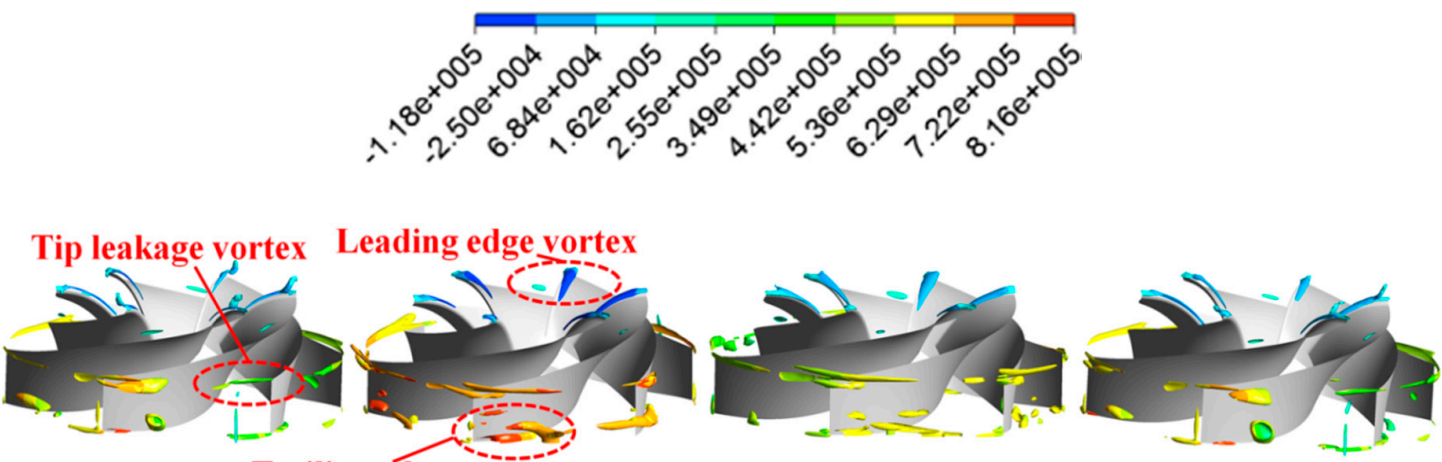

Trailing edge vortex

Scheme 1

Scheme 2

Scheme 3

Scheme 4

(a) Vortex spatial structure at $0.2 Q_{\mathrm{d}}$

[Pa]

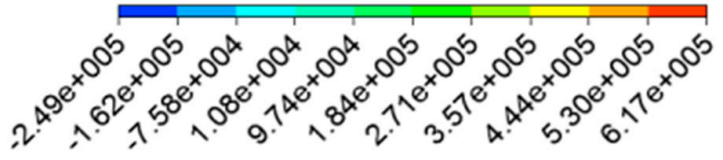

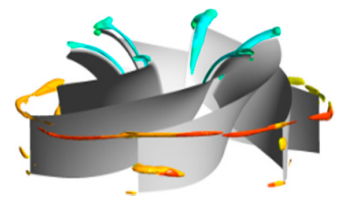

Scheme 1

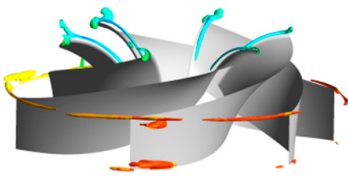

Scheme 2

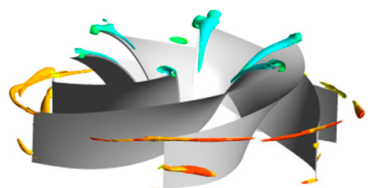

Scheme 3

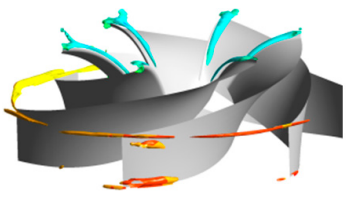

Scheme 4

(b) Vortex spatial structure at $0.6 Q_{\mathrm{d}}$

Figure 10. Cont. 
$[\mathrm{Pa}]$

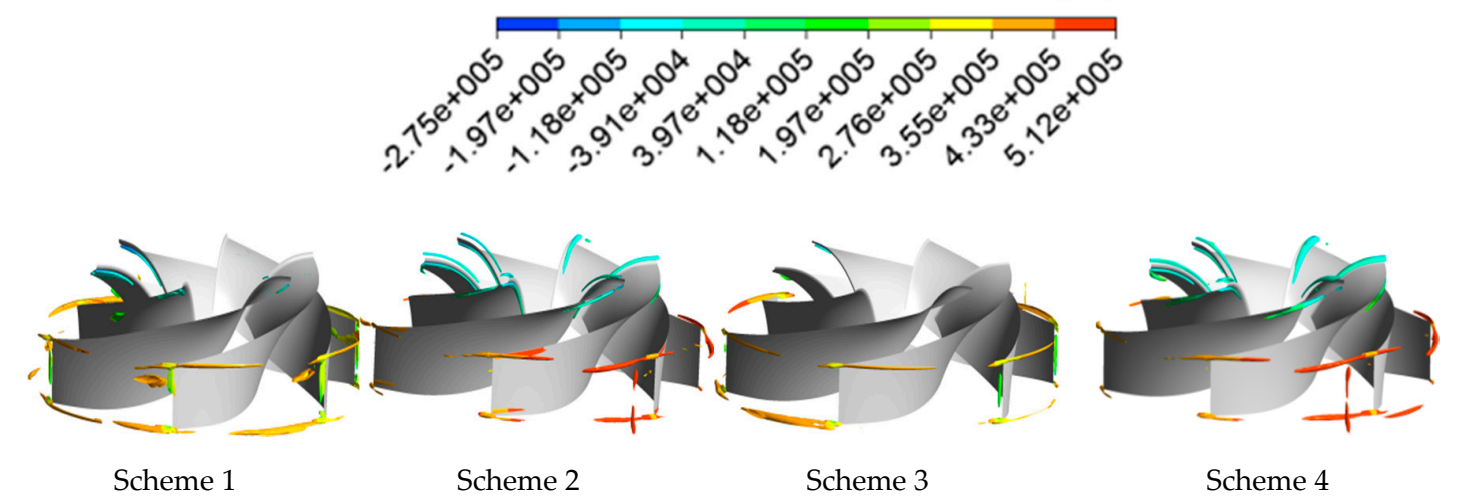

(c) Vortex spatial structure at $1.0 Q_{\mathrm{d}}$

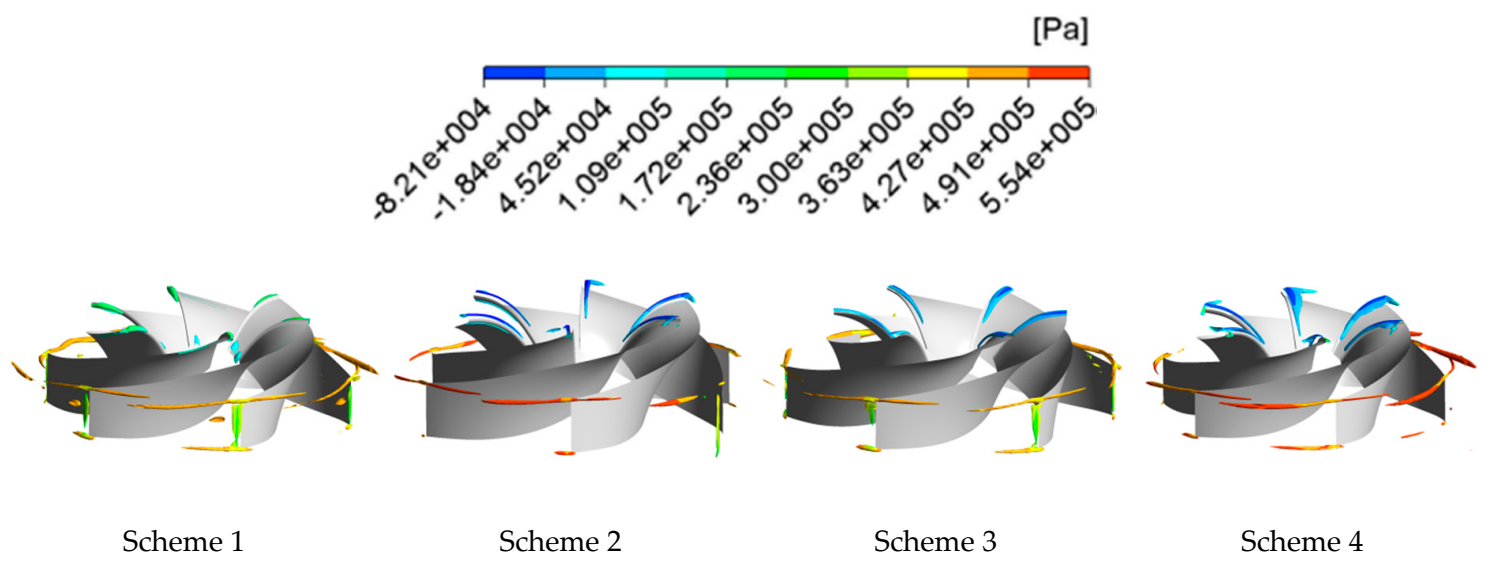

(d) Vortex spatial structure at $1.4 Q_{\mathrm{d}}$

Figure 10. 3D vortex structure for various flow conditions for four different blade thickness distributions.

As shown in Figure 10, GLV is generated at the gap between the blade and volute, which looks like a silk ribbon surrounding the entire impeller. This vortex structure is generated due to pressure difference between the pressure surface of the blade (PSB) and the suction surface of the blade (SSB), which results in water flowing from PSB to SSB through the gap and interacting with the mainstream in the impeller passage. The rotation direction of the vortex is the same as the impeller. It can also be noted that with the increase in the flow condition, the pressure difference between the PSB and SSB gradually decreases, which results in a corresponding decline in the size and strength of GLV.

Meanwhile, due to the impact of the incoming flow on the impeller, LEV can be observed at the leading edge of the blade. By comparing the LEV at various flow conditions, it can be concluded that the size and strength of LEV has a negative correlation with the flow condition; in other words, the size and strength of LEV reaches the maximum at the partial-load flow condition $\left(0.2 Q_{d}\right)$ and reaches the minimum at $1.0 Q_{d}$. The incoming flow on the impeller at the design flow condition is considered as the non-impact flow, that is, the internal flow field is smooth without any vortices. However, when the flow condition decreases from $1.0 Q_{d}$ to the $0.2 Q_{d}$, the axial velocity remains the same, but the meridional velocity gradually decreases, which results in the increase of the impeller incidence angle. Therefore, the impact of incoming flow is felt at the leading edge of the blade. When the flow condition increases from $1.0 Q_{\mathrm{d}}$ to the $1.4 Q_{\mathrm{d}}$, the incidence angle is larger than the design incidence angle which also leads to generation of LEV at leading edge of the blade. In addition, it can be noted that the negative pressure is created at the leading edges of the blades which may induce cavitation that can deteriorate the impeller. The incoming flow at the inlet of the impeller is squeezed, which a causes large 
change in the velocity direction and thus the pressure reduces sharply at the leading edge of the blade. In addition, since the expelling coefficient of the blade with thicker leading edge is larger than that with thinner leading edge, the magnitude and area of negative pressure on the thicker leading edge blade (Schemes 2 and 4) is significantly larger than that on the thinner leading edge blade (Schemes 1 and 3). Furthermore, as the flow condition increases, the area of the negative pressure gradually decreases.

At the partial-load conditions $\left(0.2 Q_{\mathrm{d}}-0.6 Q_{\mathrm{d}}\right)$, the TEV can be seen at the trailing edge of the blade, as shown in Figure 10a,b, where the shape of the TEV is like a bubble attached to the PSB. Generally, the morphology of the TEV is more disordered than that of the LEV and GLV, and the pressure and intensity of TEV are significantly higher than for the other two vortices. As the flow condition continues to increase, the intensity of the TEV gradually declines. Furthermore, it can be observed that the TEV of the Scheme 2 is the most disorderly one, which has a twisted shape. This not only effects the flow movement of the impeller passage, but also decreases the hydraulic performance. Therefore, based on these observations on vortex structures, it can be concluded that the characteristics of the TEV are more unstable than those of LEV and GLV.

To further investigate the formation principles of the unsteady vortex in detail, the vorticity transport equation $[20,21]$ is used in this research:

$$
\frac{\vec{\omega}}{D t}=(\vec{\omega} \cdot \nabla) \vec{V}-\vec{\omega}(\nabla \cdot \vec{V})+\frac{\nabla \rho_{m} \times \nabla p}{\rho_{m}^{2}}+v \nabla^{2} \vec{\omega}
$$

In Equation (7), $D \vec{\omega} / D t$ is the rate of the vorticity variation, $(\vec{\omega} \cdot \nabla) \vec{V}$ is the vortex stretching variable (VSV) caused by the velocity gradients, and $\vec{\omega}(\nabla \cdot \vec{V})$ represents the vortex dilation, which reveals the influence of the medium compressibility on the vorticity. The term $\nabla \rho_{m} \times \nabla p / \rho_{m}^{2}$ is the baroclinic torque, which is induced by the gradient of both the pressure and density. $v \nabla^{2} \vec{\omega}$ is the viscidity diffusion variable (VDV), which determines the effect of viscidity diffusion on vorticity. Considering the fact that the medium is water which is incompressible, the terms $\vec{\omega}(\nabla \cdot \vec{V})$ and $\nabla \rho_{m} \times \nabla p / \rho_{m}^{2}$ are neglected in this paper.

Figure 11 shows the distribution of the viscidity diffusion variable (VDV) on the PSB for various flow conditions. It can be observed that an oval shape VDV is generated near the trailing edge of the blade, and the magnitude of the VDV gradually decreases with an increase in the flow condition. The VDV distribution reaches maximum at the trailing edge at a flow condition of $100 \mathrm{~m}^{3} / \mathrm{h}$ and it moves towards the leading edge for high flow condition of $700 \mathrm{~m}^{3} / \mathrm{h}$. Since the blade passage is full of uneven flow and axial eddies at the partial-load conditions, the induced relative velocity on the PSB is smaller than the ideal design relative velocity. The separation of the flow in the boundary layer occurs due to the effect of the positive incidence angle. The conclusion can be drawn that the thicker the blade is, the greater the flow separation becomes. Furthermore, when the flow condition reaches the design value, the VDV distribution reduces to its minimum. And at an over-load condition $\left(700 \mathrm{~m}^{3} / \mathrm{h}\right)$, a larger VDV is generated at the leading edge.

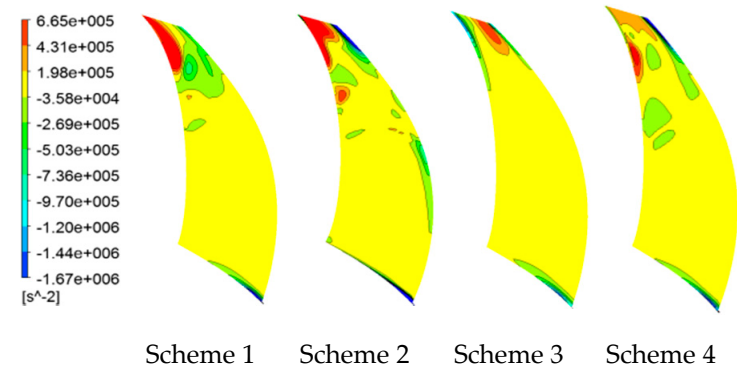

(a) The viscidity diffusion variable (VDV)distribution at $100 \mathrm{~m}^{3} / \mathrm{h}$
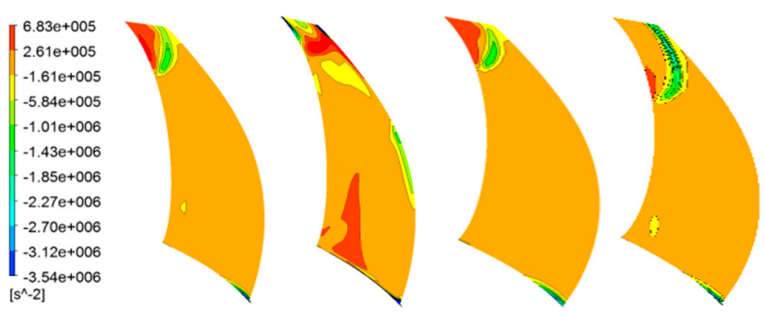

Scheme 1 Scheme 2

Scheme 3

Scheme 4

(b) The VDV distribution at $300 \mathrm{~m}^{3} / \mathrm{h}$

Figure 11. Cont. 

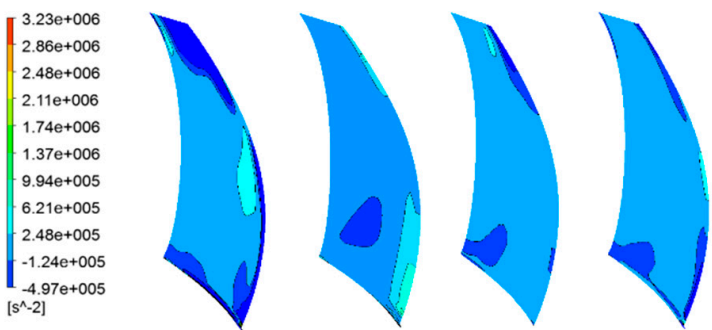

Scheme 1 Scheme 2 Scheme $3 \quad$ Scheme 4

(c) The VDV distribution at $500 \mathrm{~m}^{3} / \mathrm{h}$
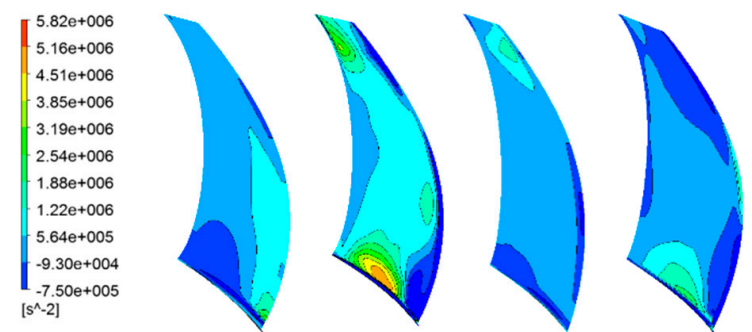

Scheme $1 \quad$ Scheme $2 \quad$ Scheme $3 \quad$ Scheme 4

(d) The VDV distribution at $700 \mathrm{~m}^{3} / \mathrm{h}$

Figure 11. The VDV distribution at various flow conditions.

The vortex Stretching Variable (VSV) distribution is similar to the VDV distribution, as shown in Figure 12. The VSV distribution is based on the angular momentum conservation principle [22]. VSV first reduces and then gradually increases; the lowest value of VSV is obtained at the design condition. Based on this discussion, it can be concluded that the VSV plays an essential role in the formation of the vortex, and more importantly that increasing the thickness of the blade is an effective way to decrease the VSV.
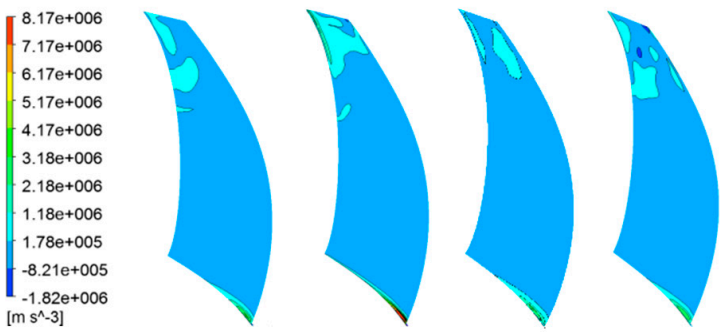

Scheme 1

Scheme 2

Scheme 3

Scheme 4

(a) The Vortex Stretching Variable (VSV)distribution at

$\left[\begin{array}{l}3.63 e+005 \\ -1.62 e+005 \\ -3.96 e+004 \\ -2.41 e+005 \\ -4.42 e+005 \\ -6.44 e+005 \\ -8.45 e+005 \\ -1.05 e+006 \\ -1.25 e+006 \\ -1.45 e+006 \\ -1.65 e+006 \\ {\left[\mathrm{~m} \mathrm{~s}^{\wedge}-3\right]}\end{array}\right.$

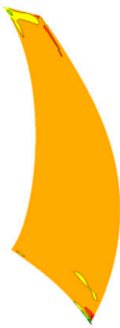
$100 \mathrm{~m}^{3} / \mathrm{h}$

Scheme $1 \quad$ Scheme $2 \quad$ Scheme $3 \quad$ Scheme 4 (c) The VSV distribution at $500 \mathrm{~m}^{3} / \mathrm{h}$
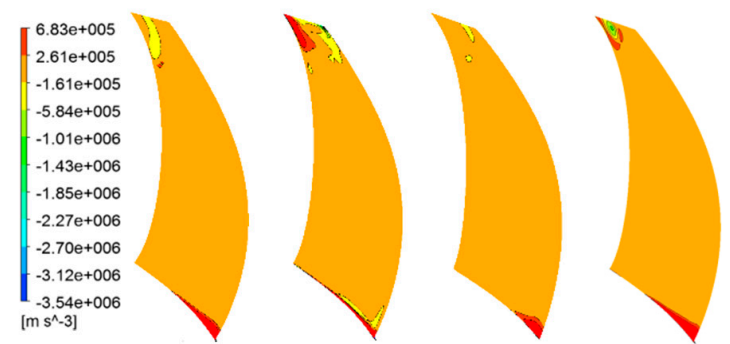

Scheme 1

Scheme 2

Scheme 3

Scheme 4

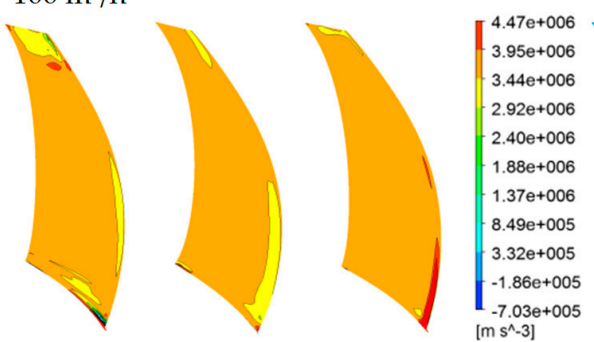

(b) The VSV distribution at $300 \mathrm{~m}^{3} / \mathrm{h}$
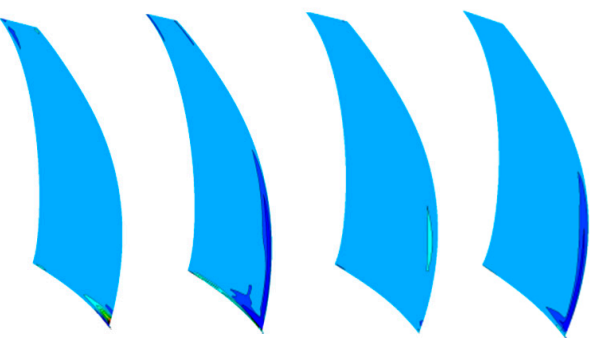

Scheme $1 \quad$ Scheme $2 \quad$ Scheme $3 \quad$ Scheme 4

(d) The VSV distribution at $700 \mathrm{~m}^{3} / \mathrm{h}$

Figure 12. The VSV distribution at various flow conditions.

\subsection{Energy Characteristic of the Pump}

The self-priming pump consumes and converts energy during its operation. Noting that the entropy always increases in the irreversible thermal processes, the entropy production is calculated to evaluate the energy loss of the pump. The total entropy production can be obtained as follows [15]:

$$
S_{\text {Total }}=S_{H}+S_{V}+S_{T}+S_{W}
$$

In Equation (8), $S_{H}$ represents the entropy production caused by the heat conversion. Since the self-priming pump operates under the condition of a constant temperature, this term is neglected in this study. $S_{W}$ denotes the entropy production due to the dissipation of kinetic energy and potential energy on the wall (WEP). $S_{V}$ is the entropy production from the viscidity diffusion (VEP) and $S_{T}$ is 
the entropy production generated by the turbulence which can determined by the loss in turbulent kinetic energy (TEP). The rate of the entropy production is obtained as follows: [12]:

The rate of entropy production from VEP can be expressed as:

$$
\mathrm{s}_{v}=\frac{\mu}{T}\left[2\left\{\left(\frac{\partial \bar{u}}{\partial x}\right)^{2}+\left(\frac{\partial \bar{v}}{\partial y}\right)^{2}+\left(\frac{\partial \bar{w}}{\partial z}\right)^{2}\right\}+\left(\frac{\partial \bar{u}}{\partial y}+\frac{\partial \bar{v}}{\partial x}\right)^{2}+\left(\frac{\partial \bar{u}}{\partial z}+\frac{\partial \bar{w}}{\partial x}\right)^{2}+\left(\frac{\partial \bar{v}}{\partial z}+\frac{\partial \bar{w}}{\partial y}\right)^{2}\right]
$$

where $\mu$ is the dynamic viscosity and $T$ is the temperature.

The rate of entropy production from VEP can be expressed as:

$$
\left.\mathrm{s}_{t}=\frac{\mu}{T}\left[2, \overline{\left(\frac{\partial u^{\prime}}{\partial x}\right)^{2}}+\overline{\left(\frac{\partial v^{\prime}}{\partial y}\right)^{2}}+\overline{\left(\frac{\partial w^{\prime}}{\partial z}\right)^{2}}\right\}+\overline{\left(\frac{\partial u^{\prime}}{\partial y}+\frac{\partial v^{\prime}}{\partial x}\right)^{2}}+\overline{\left(\frac{\partial u^{\prime}}{\partial z}+\frac{\partial w^{\prime}}{\partial x}\right)^{2}}+\overline{\left(\frac{\partial v^{\prime}}{\partial z}+\frac{\partial w^{\prime}}{\partial y}\right)^{2}}\right]
$$

Since $S_{T}$ cannot be solved directly, the rate of the TEP can obtained as follows [13]:

$$
s_{t}=\frac{\rho \varepsilon}{T}
$$

where $\rho$ is the density of water $\left(1000 \mathrm{~kg} / \mathrm{m}^{3}\right)$ and $\varepsilon$ is the turbulence dissipation.

It should be noted that due to the velocity gradient on the wall, and the dissipation of kinetic and potential energy occurs during the water flow between the turbulent layer and the laminar layer. Most of the previous work in this area has neglected the energy loss of the WEP. In this paper, the rate of WEP is calculated by the expression [23]:

$$
s_{w}=\frac{\tau_{w} v_{w}}{T}
$$

The WEP on the wall is obtained as:

$$
S_{W}=\int_{A} \frac{\tau_{w} v_{w}}{T} d A
$$

where $\tau_{w}$ represents the shear stress of the wall, while $v_{w}$ is the speed of the first fluid layer near the wall. Thus, the VEP and TEP generated in the fluid region can be obtained as follows:

$$
\begin{aligned}
& s_{T}=\int_{V} s_{t} d V \\
& s_{V}=\int_{V} s_{v} d V .
\end{aligned}
$$

Figure 13 shows the entropy production of the impeller for various flow conditions. It can be observed that the TEP of the blades with a thick leading edge (Schemes 2 and 4) is larger than that of the blades with a thin leading edge (Schemes 1 and 3). Furthermore, the TEP of the blade with increasing thickness from the leading edge to the middle section and trailing edge (Scheme 3 ) is lower than that of the uniformly thin blade (Scheme 1). As the flow condition gradually increases, the TEP first decreases drastically but then smoothly increases at the design flow condition. In addition, the decrement rate of TEP at partial-load condition is larger than the growth rate under the over-load condition, and the TEP at design flow condition drops down to the minimum value. By comparing the VEP and WEP for different blade thicknesses, it can be observed that their variation trends are consistent with TEP; however, both the VEP and WEP of Scheme 2 are higher than those of other schemes. Furthermore, as the flow condition increases, both the VEP and WEP continue to rise but the growth rate of VEP is larger than that of WEP. More importantly, comparing the magnitudes of TEP, VEP, and WEP, it can be concluded that the effects of TEP and VEP on energy loss are stronger than that of WEP. In other words, the energy loss caused by VEP and TEP are the dominant factors influencing the energy conversion in the novel self-priming pump. 


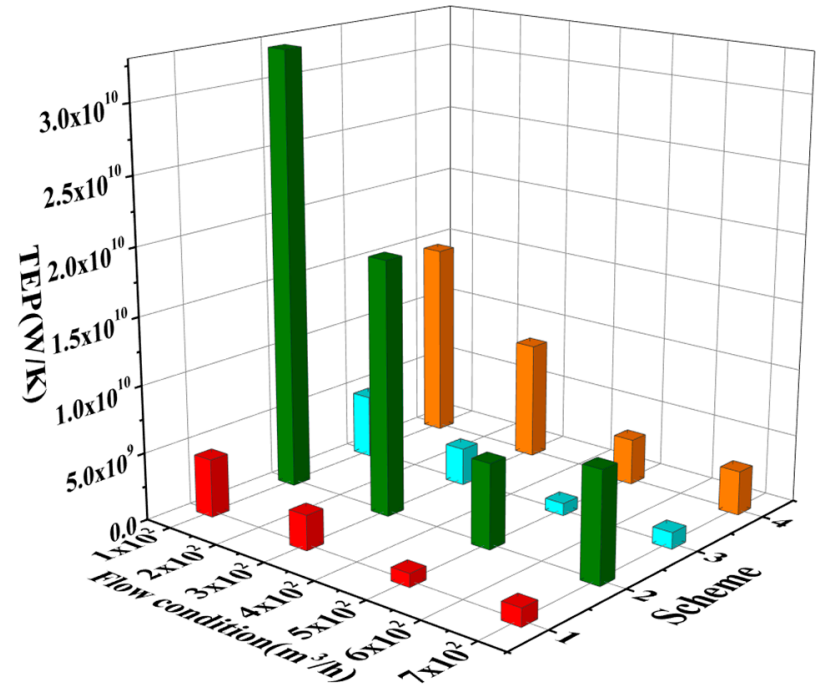

(a) The turbulence entropy production (TEP) of the impeller at various flow conditions

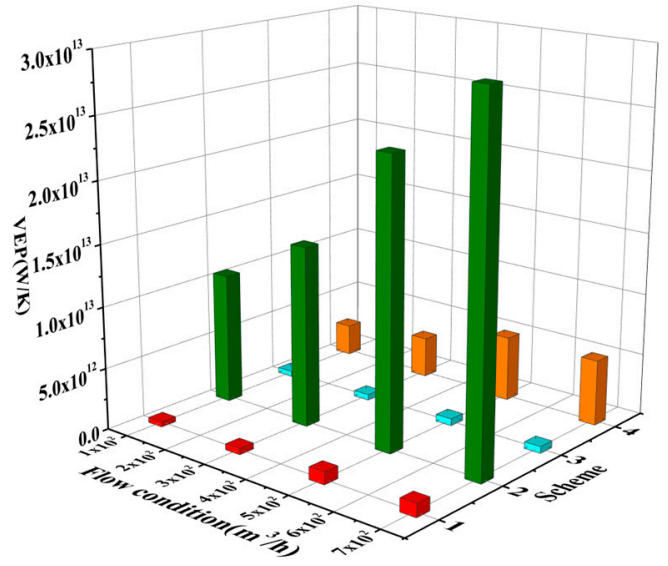

(b) The viscous entropy production (VEP) of the impeller at various flow conditions

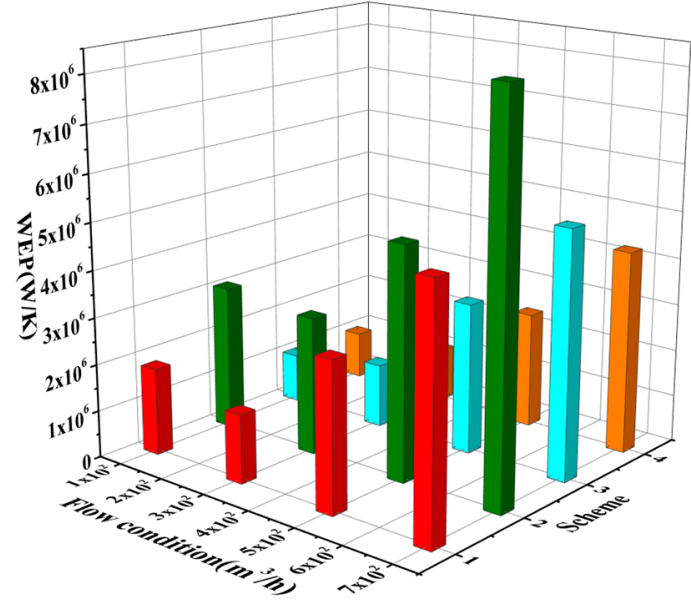

(c) The entropy production due to the dissipation of kinetic energy and potential energy on the wall (WEP) of the impeller at various flow conditions.

Figure 13. Impeller entropy production at various flow conditions. 
As shown in Figure 14, the distribution of the VEP rate coincides with the VDV distribution rate. It can be noticed that the energy loss caused by the VEP rate is mainly concentrated near the trailing edge for a partial-load condition. There is a negative correlation between the VEP and flow conditions in the range $0.2 Q_{d}$ to $1.0 Q_{d}$. This means the magnitude of the VEP rate gradually decreases when the flow condition increases to the design flow condition. On the other hand, as the flow condition further increases beyond the design flow condition, the VEP rate shows an increasing trend and the location of energy loss changes from the trailing edge to the leading edge of the blade. These results can be attributed to the change in VEP due to viscous diffusion; greater viscous diffusion can be found at the leading edge under partial-load conditions, which leads to separation of the boundary layer. Moreover, the area and magnitude of viscous diffusion in Scheme 3 are obviously lower than those in Scheme 2; in other words, utilizing the blade with increasing thickness from the leading edge (Scheme 3) is a good solution to reduce the boundary layer separation and its effect.
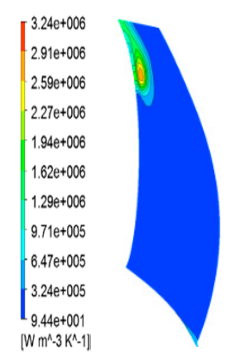

Scheme 1

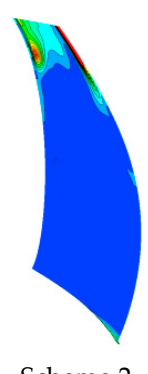

Scheme 2
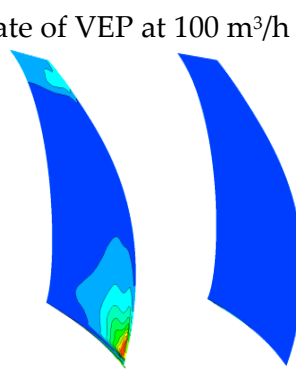

Scheme 1 Scheme 2 Scheme 3

(c) Rate of VEP at $500 \mathrm{~m}^{3} / \mathrm{h}$
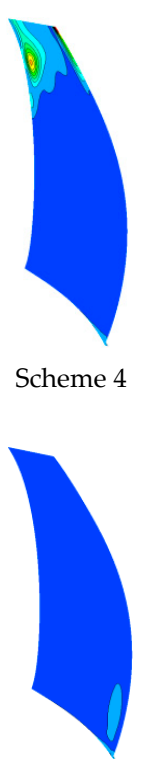

Scheme 4

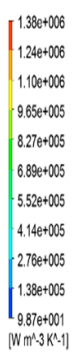
$\left[\begin{array}{l}4.06 e+005 \\ 3.66 e+005 \\ -3.25 e+005 \\ 2.844+005 \\ 2.44 e+005 \\ 2.03 e+005 \\ 1.63 e+005 \\ -1.22 e+005 \\ 8.13 e+004 \\ 4.07 e+004 \\ 5.55 e+001 \\ {\left[W m^{2}-3 k^{\wedge}-1\right]}\end{array}\right.$

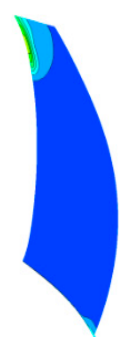

Scheme 1

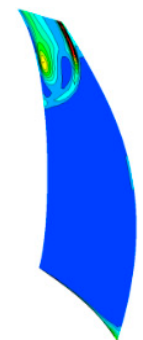

Scheme 2

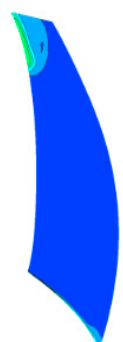

(b) Rate of VEP at $300 \mathrm{~m}^{3} / \mathrm{h}$

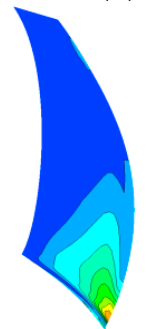

Scheme 3

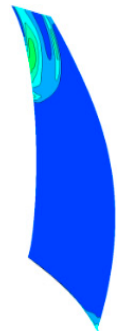

Scheme 4
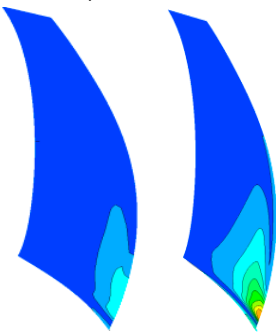

Scheme 4

Figure 14. Rate of VEP at various flow conditions [24].

Figure 15 presents the distribution of the TEP rate at various flow conditions. It can be noticed that the TEP rate distribution has a consistent variation trend with the VEP rate, but its intensity is relatively smaller. Since the TEV is created under partial-load conditions, it can not only reduce the flow area of the impeller but also can induce turbulent dissipation at the trailing edge. In addition, by comparing the TEP rates of each schemes, it can be found that a larger TEP rate occurs at the trailing edge of the thick blade (Schemes 2 and 4), and that the TEP of Scheme 3 is closer to the ideal flow field.

Thus, based on the above observations, it can be concluded that the flow condition creates negative effect on the TEP and VEP rate in the range $0.2 Q_{\mathrm{d}}$ to $1.0 Q_{\mathrm{d}}$, and the lowest TEP and VEP rates are obtained for $1.0 Q_{\mathrm{d}}$. Furthermore, as the flow condition increases to the over-load condition, the TEP and VEP rates gradually increase and the energy loss caused by the VEP and TEP decrease to the minimum of the design condition. In addition, the entropy production from the thick blade is larger than that from the thin blade. Therefore, it can be concluded that the blade thickness distribution plays an essential role in the energy loss of the self-priming pump. 


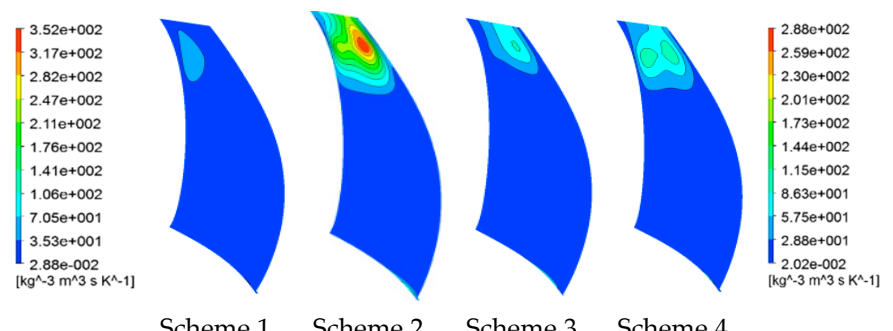

(a) Rate of TEP at $100 \mathrm{~m}^{3} / \mathrm{h}$

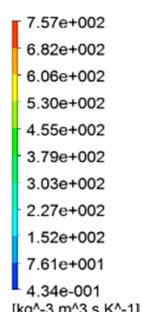

Scheme 1 Scheme 2 Scheme 3 Scheme 4

(c) Rate of TEP at $500 \mathrm{~m}^{3} / \mathrm{h}$
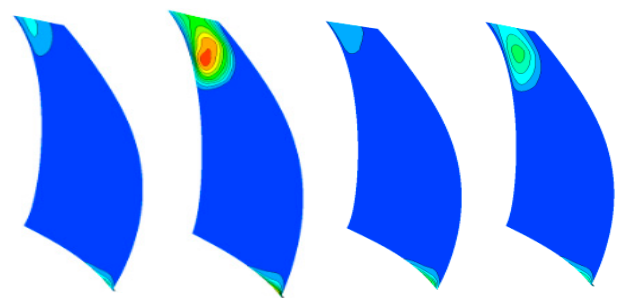

Scheme 1

Scheme 2

Scheme 3

Scheme 4

(b) Rate of TEP at $300 \mathrm{~m}^{3} / \mathrm{h}$
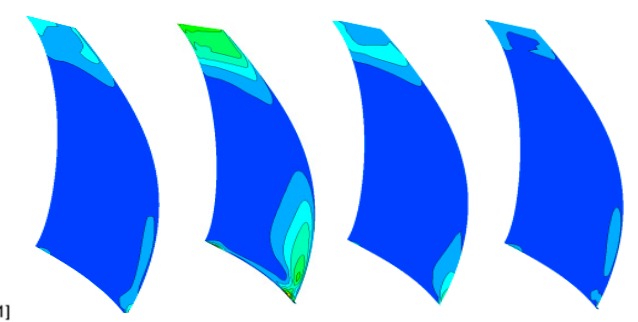

Scheme $1 \quad$ Scheme 2 Scheme $3 \quad$ Scheme 4

(d) Rate of TEP at $700 \mathrm{~m}^{3} / \mathrm{h}$

Figure 15. Rate of TEP at various flow conditions [24].

\section{Hydraulic Performance of the Pump}

To compare the hydraulic performance of the pump with different thicknesses of the blade (Schemes 1-4), the head and efficiency are selected as the two key quantities for evaluating its hydraulic characteristics. The head indicates the hoisting height of water when it receives energy from the pump, while the efficiency expresses the energy conversion ability of the self-priming pump. The head and efficiency are defined as:

$$
H=\frac{P_{O}-P_{I}}{\rho g}+\frac{V_{O}^{2}-V_{I}^{2}}{2 g}+\left(Z_{O}-Z_{I}\right)
$$

and

$$
\eta=\frac{60 g H Q_{F}}{\pi M n}
$$

where $P_{O}-P_{I}, V_{O}^{2}-V_{I}^{2}$ and $Z_{O}-Z_{I}$ denote the pressure difference, velocity difference, and height difference obtained when the water flow through the pump, $M$ is the torque, and $Q_{\mathrm{F}}$ is the flow conditions of the self-priming pump. In the analysis and presentation of results, the head and flow conditions are converted into the dimensionless parameters given below [24]:

$$
\begin{gathered}
\Phi=\frac{60 Q_{F}}{\pi^{2} D_{2} b_{2} n} \\
\Psi=\frac{7200 H g}{\pi^{2} D_{2}^{2} n^{2}} .
\end{gathered}
$$

Figure 16 shows the differences in hydraulic performance of different schemes. It shows the formation of energy loss in the partial-load condition and over-load condition, which becomes minimal in the design condition. This trend in Figure 16 can be mainly attributed to the generation of TEV under a part-load condition and LEV under an over-load condition; however, the energy loss in the partial-load condition is more severe than in the over-load condition. Furthermore, it can be noted that the head of the Scheme 2 is lower than that of Scheme 1 overall except under the partial-load condition. Since a large TEV is generated in Scheme 2, the flow area reduces sharply, which results in large changes in the internal flow. Due to the effect of incoming flow, the head increases but results in large energy dissipation as shown in Figure 16b. Thus, the efficiency of Scheme 2 is obviously lower than that of 
Scheme 1 for flow condition of $0.6 Q_{d}$. Although the energy loss of the thinner blades (Scheme 1) is lower, its energy conversion ability is weaker than that of the blade with thicker trailing edge. More importantly, by comparing the efficiency of each scheme, it can be concluded that the energy conversion ability of Scheme 3 is superior to that of other schemes. Due to the advantages of the thin leading edge and thick trailing edge, Scheme 3 can not only significantly reduce the impact of the incoming flow in over-load condition, but also can promote the work capability of the blade on the fluid.

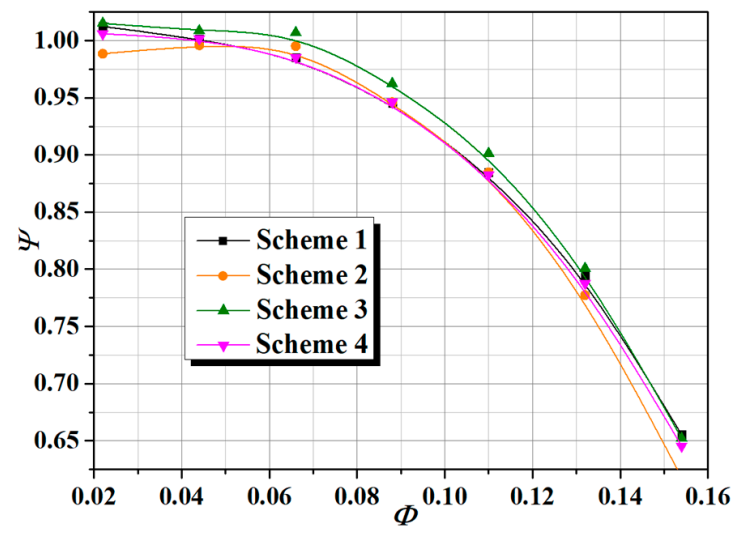

(a) Comparison of the head

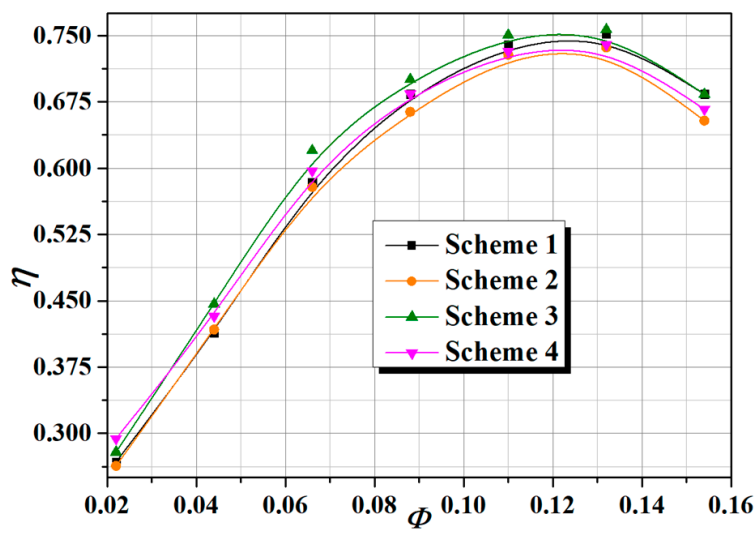

(b) Comparison of the efficiency

Figure 16. Head and efficiency under various flow conditions.

\section{Experiment Results}

To verify the accuracy of the calculations reported in Section 5, the hydraulic performance experiment for Scheme 3 (since it was found to be the best scheme in Section 5) was conducted at Jiangsu University in China. The measurement deviation of the pressure transducer $\left(D_{\mathrm{P}}\right)$ is $\pm 0.5 \%$. Considering that the monitoring points were set on the inlet and outlet tank and the measurement deviation of dynamic head can be ignored $\left(D_{\mathrm{D}}=0\right)$, the measurement deviation of head $\left(D_{\mathbf{H}}\right)$ is obtained as follows:

$$
D_{\mathrm{H}}=\sqrt[2]{D_{\mathrm{P}}^{2}+D_{\mathrm{D}}^{2}}
$$

Furthermore, the deviation of the transducer turbine flowmeter $\left(D_{\mathrm{Q}}\right)$ is $\pm 1.0 \%$, the measurement deviations of torque $\left(D_{\mathrm{M}}\right)$ and speed transducer $\left(D_{\mathrm{N}}\right)$ are $\pm 0.5 \%$ and $\pm 0.1 \%$, respectively. Therefore, the measurement deviation of shaft power $\left(D_{\mathrm{T}}\right)$ can be determined by:

$$
D_{\mathrm{T}}=\sqrt[2]{D_{\mathrm{N}}^{2}+D_{\mathrm{M}}^{2}}
$$

Finally, the measurement deviation of experimental system $\left(D_{\mathrm{S}}\right)$ can be expressed as follows:

$$
D_{\mathrm{S}}=\sqrt[2]{D_{\mathrm{H}}^{2}+D_{\mathrm{Q}}^{2}+D_{\mathrm{T}}^{2}}
$$

Therefore, the measurement deviation of the experiment is within $\pm 1.22 \%$ which quantifies the uncertainty in experimental measurements. The experimental results are shown in Figure 17. It can be seen from this figure that overall the experimental and numerical results for pump head and efficiency are in reasonable agreement with minor discrepancies. It should be noted that in the calculations, the internal structure of the pump was simplified. This meant the pump cavity, seal, and bearing were not considered in the numerical model. As a result, the pump performance results obtained in the calculations are slightly larger than those in the experiment. However, the maximum discrepancy between the numerical results and experimental results is within $3 \%$, which is in the acceptable range. It can therefore be concluded that the numerical model and computations reported in this paper are accurate and the results are reliable. 


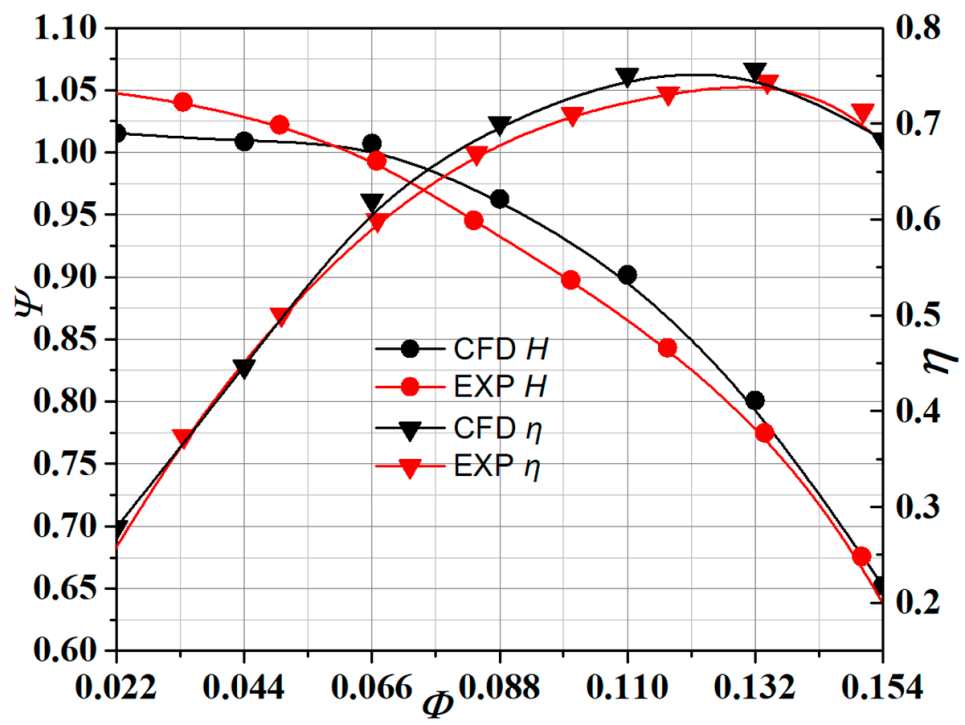

Figure 17. Comparison of experimental and numerical results for pump head and efficiency for Scheme 3.

In addition, to validate the capability of a novel self-priming pump, the experiments with waterless start were conducted at different altitudes at Jiangsu University in China.

Figure 18 shows the results of self-priming pump experiment at $5.3 \mathrm{~m}, 6 \mathrm{~m}, 7 \mathrm{~m}$, and $8 \mathrm{~m}$ altitudes. It should be noted that the self-priming time reaches $62 \mathrm{~s}$ at $5.3 \mathrm{~m}$ altitude, which is remarkably shorter than the standard Chinese self-priming pump time of $120 \mathrm{~s}$. Furthermore, it can be noted from Figure 18 that the increase rate in self-priming time $R$ with the altitude can be separated into two stages, namely the altitude from $0 \mathrm{~m}$ to $6 \mathrm{~m}$ and from $6 \mathrm{~m}$ to $8 \mathrm{~m}$. The first stage shows the non-linear variation while the second stage shows the linear variation in start-up time with the altitude. When the self-priming altitude is below $6 \mathrm{~m}$, with increase in the altitude more resistance of water needs to be overcome, therefore negative pressure is generated in the pump which induces the air in the water to separate and leads to the air in the pump increasing. Thus, the line of the increase rate $R$ in Figure 18 shows a nonlinear increase. On the other hand, as the altitude continues to increase, the air in the water is no longer discharged. Therefore, the relationship between air volume in the pump and altitude shows a linear increase, which is the reason for the linear growth in the second stages.

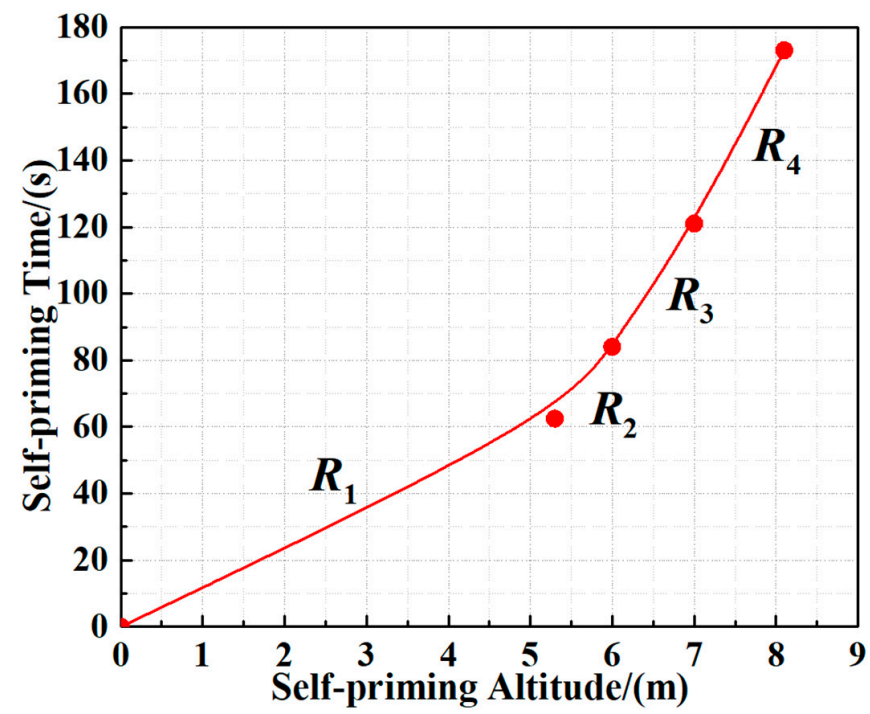

Figure 18. Self-priming time at different altitudes. 


\section{Conclusions}

In this paper, by using the $Q$ criterion with vorticity transport equation and analyzing the entropy production in the flow, the vortex structures and energy characteristics of a novel self-priming pump with different blade thicknesses were systematically investigated by numerical simulations which are validated for one of the most promising blade thickness distributions in terms of the pump head and efficiency. The following conclusions can be drawn.

A new self-priming pump has been designed which can achieve the pump start without water. Under the effect of the self-priming system, negative pressure is generated in the pump which results in rapid suction of the water in the pump. It is demonstrated that the self-priming capability of the pump is remarkably superior to the traditional pump.

The spatial structure of vortices in the pump is investigated by employing the $Q$ criterion with the numerical solution of the vorticity transport equation. Based on the morphology, the vortices be separated into three categories: Trailing Edge Vortex (TEV), Leading Edge Vortex (LEV), and Gap Leakage Vortex (GLV). Generally, the morphology of the TEV is more disorderly than that of LEV and GLV, and the pressure and intensity of TEV are significantly higher than the other two vortex structures. TEV cannot only block the passage of the impeller but can also increase instability in the internal flow. Analysis of the formation mechanism of the vortices using the numerical solution of the vorticity transport equation shows that the Viscous Diffusion Variable (VDV) plays an essential role in the separation of the boundary layer which results in the generation of Tip Leakage Vortex (TLV). In addition, the thicker the blade is, the more severe the flow separation becomes.

To determine the magnitude and distribution of energy loss in the pump, entropy production analysis is applied to study the influence of blade thickness on energy characteristics. Based on the analysis, greater energy loss is generated in both the partial-load condition and the over-load condition relative to design condition; however, the energy loss in partial-load condition is larger than that in the over-load condition due to differences in the size and intensity of TLV. Furthermore, in other words, the energy loss caused by viscous entropy production (VEP) and turbulence entropy production (TEP) are the dominant factors influencing the energy loss in the novel self-priming pump. The energy loss of the thicker blade is larger than that of the thinner blade; however, employing the blade with a thin leading edge and thick trailing edge can not only significantly reduce the impact of incoming flow under over-load condition but can also increase the work capability of the blade on the fluid. Thus, increase in thickness of the blade from the leading edge to the trailing edge is beneficial for pump performance.

Finally, the hydraulic test confirmed the accuracy of the simulations. Therefore, the numerical results presented in this paper can be considered as accurate and reliable.

Author Contributions: This is a joint work and the authors contributed according to their expertise and capability: H.C. conducted the investigation and analysis, and writing and revisions of the paper; R.K.A. ontributed to methodology and revisions of the paper; W.L. Performed the validation and revision; L.Z. Performed the data analysis and W.S. was involved in revision of the manuscript.

Funding: This work was sponsored by the National Natural Science Foundation of China (No. 51979138, No. 51679111, No. 51409127 and No. 51579118), National Key R\&D Program Project (No. 2017YFC0403703), PAPD, and Six Talents Peak Project of Jiangsu Province (No. JNHB-CXTD-005), Key R\&D Program Project in Jiangsu Province (BE2015119, BE2015001-4, BE2016319, BE2017126), Natural Science Foundation of Jiangsu Province (No. BK20161472), Science and Technology Support Program of Changzhou (No. CE20162004) and Graduate Student Scientific Research Innovation Projects of Jiangsu Province (Grant No. SJKY19_2547). The first author would like to thank Chinese Scholarship Council (CSC) for the financial support and Washington University in St. Louis for providing the opportunity to spend a year as a visiting PhD student (201808320259).

Conflicts of Interest: The authors declare that there is no conflict of interest regarding the publication of this paper.

\section{Nomenclature}

$H \quad$ head $\mathrm{m}$

$Q_{\mathrm{d}} \quad$ design flow condition, $\mathrm{m}^{3} / \mathrm{h}$

$n \quad$ rotation speed, $\mathrm{r} / \mathrm{min}$ 


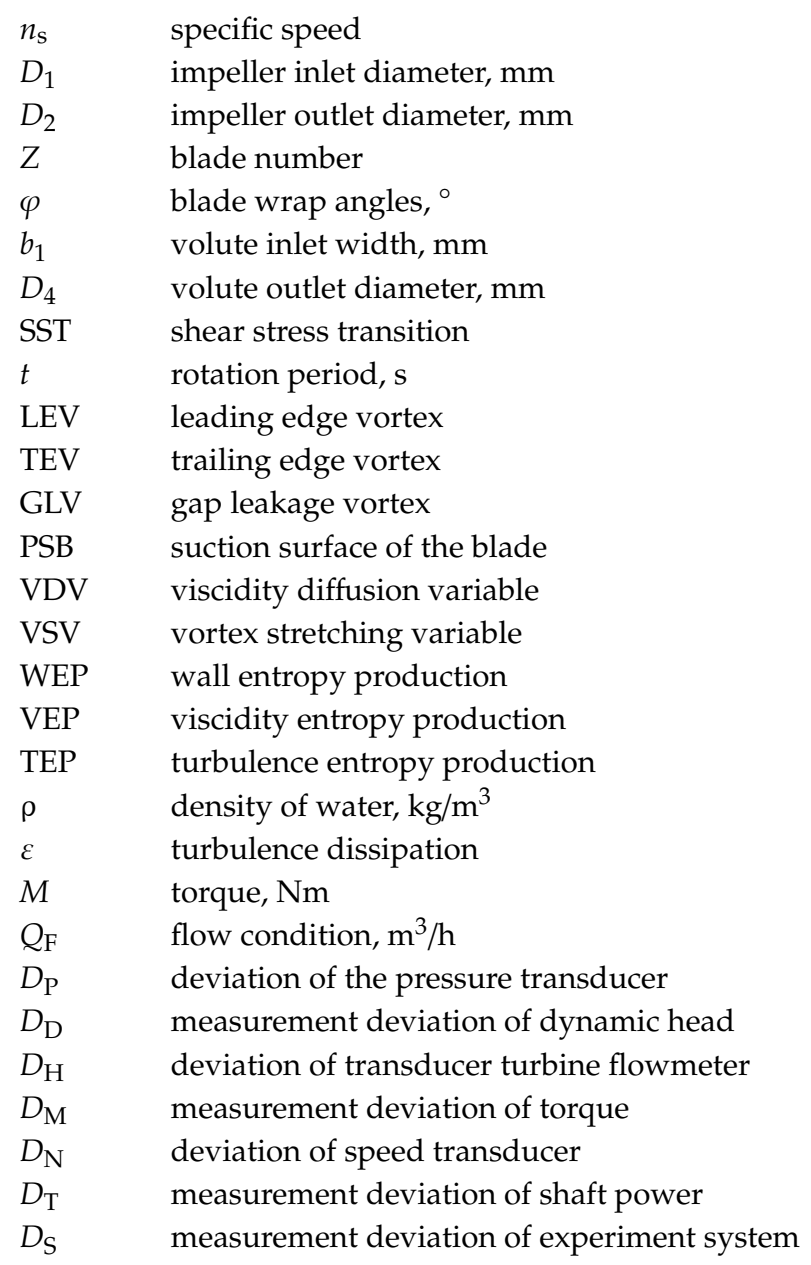

\section{References}

1. Turkyilmazoglu, M. Performance of direct absorption solar collector with nanofluid mixture. Energy Convers. Manag. 2016, 114, 1-10. [CrossRef]

2. Wang, T.; Kong, F.; Xia, B.; Bai, Y.; Wang, C. The method for determining blade inlet angle of special impeller using in turbine mode of centrifugal pump as turbine. Renew. Energy 2017, 109, 518-528. [CrossRef]

3. Elyamin, G.R.H.A.; Bassily, M.A.; Khalil, K.Y.; Mohamed, S.G. Effect of impeller blades number on the performance of a centrifugal pump. Alex. Eng. J. 2019, 58, 39-48. [CrossRef]

4. Shao, C.; Zhou, J.; Cheng, W. Effect of viscosity on the external characteristics and flow field of a molten salt pump in the view of energy loss. Heat Mass Transf. 2019, 55, 711-722. [CrossRef]

5. Nejad, J.; Riasi, A.; Nourbakhsh, A. Efficiency improvement of regenerative pump using blade profile modification: Experimental study. Proc. Inst. Mech. Eng. Part E J. Process Mech. Eng. 2019, 233, 448-455. [CrossRef]

6. Han, X.; Kang, Y.; Li, D.; Zhao, W. Impeller optimized design of the centrifugal pump: A numerical and experimental investigation. Energies 2018, 11, 1444. [CrossRef]

7. Jeon, S.Y.; Yoon, J.Y.; Jang, C.M. Optimal design of a novel 'S-shape' impeller blade for a microbubble pump. Energies 2019, 12, 179. [CrossRef]

8. Nejad, J.; Riasi, A.; Nourbakhsh, A. Parametric study and performance improvement of regenerative flow pump considering the modification in blade and casing geometry. Int. J. Numer. Methods Heat Fluid Flow 2017, 27, 1887-1906. [CrossRef]

9. Wang, C.; Hu, B.; Zhu, Y.; Wang, X.; Luo, C.; Cheng, L. Numerical study on the gas-water two-phase flow in the self-priming process of self-priming centrifugal pump. Processes 2019, 7, 330. [CrossRef]

10. Wang, C.; He, X.; Zhang, D.; Hu, B.; Shi, W. Numerical and experimental study of the self-priming process of a multistage self-priming centrifugal pump. Int. J. Energy Res. 2019, 1-19. [CrossRef] 
11. Wang, C.; He, X.; Shi, W.; Wang, X.; Wang, X.; Qiu, N. Numerical study on pressure fluctuation of a multistage centrifugal pump based on whole flow field. AIP Adv. 2019, 9, 035118. [CrossRef]

12. Wang, C.; Zhang, Y.; Hou, H.; Zhang, J.; Xu, C. Entropy production diagnostic analysis of energy consumption for cavitation flow in a two-stage LNG cryogenic submerged pump. Int. J. Heat Mass Transf. 2019, 129, 342-356. [CrossRef]

13. Hou, H.; Zhang, Y.; Zhou, X.; Zuo, Z.; Chen, H. Optimal hydraulic design of an ultra-low specific speed centrifugal pump based on the local entropy production theory. Proc. Inst. Mech. Eng. Part A J. Power Energy 2019, 233, 715-726. [CrossRef]

14. Gu, Y.; Pei, J.; Yuan, S.; Wang, W.; Zhang, F.; Wang, P.; Appiah, D.; Liu, Y. Clocking effect of vaned diffuser on hydraulic performance of high-power pump by using the numerical flow loss visualization method. Energy 2019, 170, 986-997. [CrossRef]

15. Li, D.; Wang, H.; Qin, Y.; Han, L.; Wei, X.; Qin, D. Entropy production analysis of hysteresis characteristic of a pump-turbine model. Energy Convers. Manag. 2017, 149, 175-191. [CrossRef]

16. Chang, H.; Li, W.; Shi, W.; Liu, J. Effect of blade profile with different thickness distribution on the pressure characteristics of novel self-priming pump. J. Braz. Soc. Mech. Sci. Eng. 2018, 40, 518. [CrossRef]

17. Bai, L.; Zhou, L.; Han, C.; Zhu, Y.; Shi, W. Numerical study of pressure fluctuation and unsteady flow in a centrifugal pump. Processes 2019, 7, 354. [CrossRef]

18. Bai, L.; Zhou, L.; Jiang, X.; Pang, Q.; Ye, D. Vibration in a multistage centrifugal pump under varied conditions. Shock Vib. 2019, 2057031. [CrossRef]

19. Alfonsi, G.; Primavera, L. Temporal evolution of vortical structures in the wall region of turbulent channel flow. Flow Turbul. Combust. 2009, 83, 61-79. [CrossRef]

20. Ji, B.; Luo, X.; Arndt, R.E.A.; Wu, Y. Numerical simulation of three-dimensional cavitation shedding dynamics with special emphasis on cavitation-vortex interaction. Ocean Eng. 2014, 87, 64-77. [CrossRef]

21. Liu, Y.; Tan, L. Tip clearance on pressure fluctuation intensity and vortex characteristic of a mixed flow pump as turbine at pump mode. Renew. Energy 2018, 129, 606-615. [CrossRef]

22. Wu, J.Z.; Ma, H.Y.; Zhou, M.D. Vorticity and Vortex Dynamic; Springer Science \& Business Media: Berlin/Heidelberg, Germany, 2007.

23. Hucan, H.; Zhang, Y.; Li, Z. A numerical research on energy loss evaluation in a centrifugal pump system based on local entropy production method. Therm. Sci. 2017, 21, 1287-1299.

24. Chang, H.; Shi, W.; Li, W.; Liu, J. Energy loss analysis of novel self-priming pump based on the entropy production theory. J. Therm. Sci. 2019, 28, 306-318. [CrossRef] 